EDITORIAL

\title{
Cardiotrophin-1 in Patients with Acute Coronary Syndromes: Does it Have a Role?
}

\author{
Humberto Villacorta ${ }^{(1)}$ \\ Universidade Federal Fluminense, Niterói, RJ - Brazil \\ Editorial referring to the article: The Correlation between Cardiac Enzymes and Cardiotrophin-1 Levels in Patients with Acute Coronary Syndrome
}

Acute coronary syndromes are among the most common diagnoses in the emergency department (ED). It has been estimated that 300,000 to 400,000 cases are seen each year in Brazil. ${ }^{1}$ The diagnosis of acute myocardial infarction (AMI) is made on the basis of symptoms, electrocardiogram, and biological markers of myocardial necrosis. Troponins are the most specific cardiac markers, and in the era of highly sensitive assays, they have been shown to make the exclusion of AMI in the ED more rapid, being particularly helpful in patients with atypical presentations. ${ }^{2}$

Cardiotrophin-1 (CT-1) is a member of the interleukin-6 superfamily of proinflammatory cytokines. It is expressed in many tissues, including heart and vessels. CT-1 is upregulated in cardiac fibroblasts and cardiomyocytes in response to mechanical, humoral, metabolic, and hypoxic stress (Figure 1) ${ }^{3}$ It is elevated in the myocardium and plasma of heart failure (HF) patients and has also been associated with hypertension, diabetes, cardiac hypertrophy, and fibrosis, both in patients and experimental studies. ${ }^{3,4} \mathrm{CT}-1$ is expressed after an AMI and seems to be involved in the healing and remodeling process that occurs after myocardial infarction. ${ }^{5,6}$ In the study by Freed et al., ${ }^{5} \mathrm{CT}-1$ was able to initiate each of the processes related to scar formation, including fibroblast migration and proliferation, and collagen synthesis. ${ }^{5}$

This new biomarker may have prognostic and therapeutic implications in clinical practice. In one

\section{Keywords}

Coronary Artery Disease; Myocardial Infarction; Electrocardiography/methods; Biomarkers; Cytokines. study, CT-1 was a predictor of death or HF after AMI. ${ }^{7}$ CT-1 and NT-proBNP predicted events independently of age, sex, previous AMI, serum creatinine, and Killip class (area under the curve of 0.62 for CT- 1 and of 0.77 for NT-proBNP). When both were used, the AUC was improved to $0.84 .^{7} \mathrm{CT}-1$ may additionally have a therapeutic role. In one experimental study, administration of CT-1 to rats seven days before the induction of AMI attenuated apoptosis and improved hemodynamics. ${ }^{8}$

In this issue of IJCS, Polat et al., ${ }^{9}$ sought to investigate the association between CT-1 and traditional markers of myocardial ischemia. ${ }^{9}$ The authors found a positive correlation of CT-1 with creatine kinase (CK), creatine kinase-MB (CK-MB), and troponin $\mathrm{T}$, at admission and also at six hours . The increases in CT-1 levels were especially higher in patients with ST-elevation myocardial infarction (STEMI). We congratulate the authors for bringing up recent data on this new biomarker and for confirming the results of previous work..$^{10}$ However, one strong limitation in this study is the absence of a control group, without myocardial ischemia. As pointed out before, CT-1 may be elevated in non-ischemic conditions. ${ }^{3,4}$ This lack of specificity limits the utility of this biomarker as a diagnostic tool for myocardial ischemia. Additionally, there is no head-to-head comparison of CT-1 with troponin, the gold standard biomarker in this setting.

New biomarkers are more than welcome and the authors are to be congratulated for bringing knowledge on CT-1. To be established in clinical practice, however, new biomarkers need to add information to traditional ones and, in this regard, studies comparing CT-1 with troponins have not been done. Until now, troponins remain the gold standard in the diagnosis of AMI. 


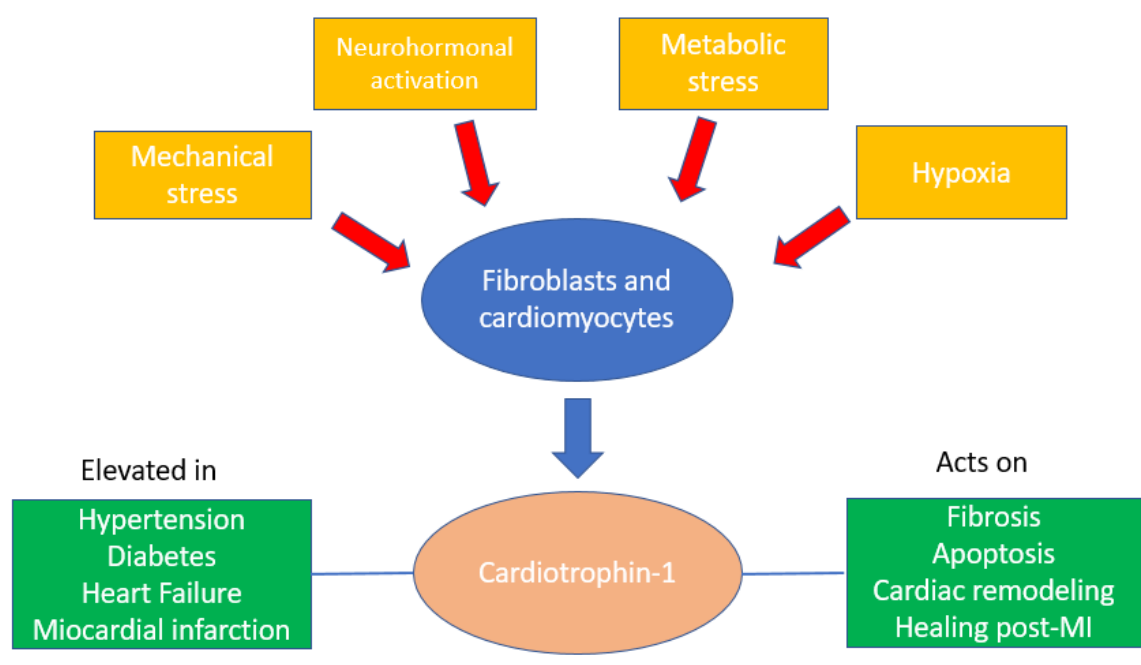

Figure 1 - Summary of known stimuli for cardiotrophin-1 upregulation in the heart and its relationship with cardiovascular diseases. MI: myocardial infarction

\section{References}

1. Brasil. Ministério da Saúde. CONITEC. Protocolo de Síndromes Coronarianas Agudas. CONITEC. [Accessed July 9th 201]. Available from:http://conitec.gov.br/images/protocolos/pcdt-sindromescoronarianas-agudas.

2. Pickering JW, Than MP, Cullen L, Aldous S, ter Avest E, Body R, et al. Rapid rule-out of acute myocardial infarction with a single highsensitivity cardiac troponin T measurement below the limit of detection. Ann Intern Med. 2017;166(10): 715-24.

3. Martinez-Martinez E, Brugnolaro C, Ibarrola J, Ravassa S, Buonafine M, López B, et al. CT-1 (cardiotrophin-1)-gal-3 (galectina-3) axis in cardiac fibrosis and inflammation: mechanistic insights and clinical implications. Hypertension. 2019; 73(3);602-11.

4. Gamela-Pozuelo L, Fuentes-Calvo I, Gómez-Marcos MA, RecioRodriguez JI, Agudo-Conde C, Fernández-Martín JL, et al. Plasma cardiotrophin-1 as a marker of hypertension and diabetes-induced target organ damage and cardiovascular risk. Medicine. 2015;94(30):1-8. Doi 10.1097/MD.0000000000001218.
5. Freed DH, Moon MC, Borowiec AM, Jones SC, Zahradka P, Dixon IMC Cardiotrophin-1: expression in experimental myocardial infarction and potential role in post-MI wound healing. Mol Cell Biochem .2003;254(1-2):247-56.

6. Freed DH, Cunnington RH, Dangerfield AL, Sutton JS, Dixon IMC Emerging evidence for the role of cardiotrophin-1 in cardiac repair in the infarcted heart. Cardiovasc Res 2005;65(4):782-92.

7. Khan SQ, Kelly D, Quinn P, Davies JE, Leong L. Cardiotrophin-1 predicts death or heart failure following acute myocardial infarction. J Card Fail 2006;12(8):635-40.

8. Ruixing Y, Dezhai Y, Jiaquan L. Effects of cardiotrophin-1 on hemodynamics and cardiomyocyte apoptosis in rats with acute myocardial infarction. J Med Invest 2004;51(1-2):29-37.

9. Polat U, Aydinlar A, Caliskan S, Boyuk F, Unal O. The correlation between cardiac enzymes and cardiotrophin-1 levels in patients with acute coronary syndrome. Int J Cardiovasc Sci. 2021;34(5Supl.1):12-21.

10. Jahromi AS, Shojaie M, Madani A. Cardiotrophin-1 in patients with acute myocardial infarction. Am J Applied Sci 2010;7(9):1190-4 